\title{
Supercorotating return flow from reconnection in Saturn's magnetotail
}

\author{
A. Masters, ${ }^{1,2}$ M. F. Thomsen, ${ }^{3}$ S. V. Badman, ${ }^{4}$ C. S. Arridge, ${ }^{1,2}$ D. T. Young, ${ }^{5}$ \\ A. J. Coates, ${ }^{1,2}$ and M. K. Dougherty ${ }^{6}$ \\ Received 10 November 2010; revised 23 December 2010; accepted 4 January 2011; published 5 February 2011.
}

[1] Detecting plasma dynamics in Saturn's magnetosphere is essential for understanding energy flow through the system. It has been proposed that both the Dungey and Vasyliunas cycles operate at Saturn, and the competition between these cycles has been debated. We examine data taken by the Cassini spacecraft in Saturn's post-dawn magnetosphere, $\sim 17.5$ Saturn radii from the planet, and identify an example of return flow from magnetotail reconnection. The flow included water group ions and had elevated ion temperatures (of order $1 \mathrm{keV}$ ), consistent with Vasyliunas cycle return flow. The flow was also supercorotating $\left(\sim 200 \mathrm{~km} \mathrm{~s}^{-1}\right.$, $\sim 120 \%$ of corotation), which is highly atypical of Saturn's outer magnetosphere. Our results suggest that return flows are time-variable, and our results concerning Dungey cycle return flows are inconclusive. We propose that supercorotating flows in Saturn's dawn magnetosphere strongly influence the current system that is responsible for the planet's main auroral emission. Citation: Masters, A., M. F. Thomsen, S. V. Badman, C. S. Arridge, D. T. Young, A. J. Coates, and M. K. Dougherty (2011), Supercorotating return flow from reconnection in Saturn's magnetotail, Geophys. Res. Lett., 38, L03103, doi:10.1029/2010GL046149.

\section{Introduction}

[2] The interaction between the solar wind and the magnetized planets produces a range of different magnetospheric systems. The energy that drives dynamics in these systems can either originate outside the magnetosphere (e.g., from the solar wind) or inside it (e.g., from the planet itself).

[3] Earth's magnetosphere, which is a slow rotator, is principally driven by the solar wind through magnetic reconnection between the planetary and interplanetary magnetic fields at the boundary of the dayside magnetosphere. This produces the Dungey cycle [Dungey, 1961], where reconnected flux tubes are transported over the poles by the solar wind, before reconnecting in the magnetotail. The

\footnotetext{
${ }^{1}$ Mullard Space Science Laboratory, Department of Space and Climate Physics, University College London, Dorking, UK.

${ }^{2}$ Centre for Planetary Sciences at UCL/Birkbeck, London, UK.

${ }^{3}$ Space Science and Applications, Los Alamos National Laboratory, Los Alamos, New Mexico, USA.

${ }^{4}$ JAXA Institute of Space and Aeronautical Science, Sagamihara, Japan.

${ }^{5}$ Space Science and Engineering Division, Southwest Research Institute, San Antonio, Texas, USA.

${ }^{6}$ Space and Atmospheric Physics Group, Blackett Laboratory, Imperial College London, London, UK.
}

Copyright 2011 by the American Geophysical Union. 0094-8276/11/2010GL046149 newly closed field lines in the tail then convect around the flanks back to the dayside, producing Dungey cycle return flow.

[4] In contrast, internal mass loading principally drives Jupiter's magnetosphere, a rapid rotator. The outward transport of mass leads to the Vasyliunas cycle [Vasyliunas, 1983], where closed field lines rotate from the dayside around the dusk flank to the nightside, and then stretch down the magnetotail. Eventually these field lines reconnect in the center of the tail, resulting in closed field lines that accelerate back to the dayside via the dawn flank, producing Vasyliunas cycle return flow.

[5] The competition between these two cycles at Saturn (a rapid rotator) is a subject of much debate. Like Jupiter, there are significant mass sources in Saturn's magnetosphere, making the operation of the Vasyliunas cycle likely. However, some authors have argued that the Dungey cycle also operates, and may be significant under certain solar wind conditions [Badman and Cowley, 2007, and references therein]. Figure 1 illustrates both Dungey-type and Vasyliunastype reconnection in Saturn's magnetotail, and the resulting return flows, which are both via the dawn flank and consist of mass-depleted flux tubes and heated plasma.

[6] Badman and Cowley [2007] discussed the competition between these two cycles at Saturn, predicting that the ion temperature of both return flows would be a few $\mathrm{keV}$, and that the different return flows could be identified on the basis of plasma composition (see Figure 1). They proposed that Dungey cycle return flow would principally contain lightions originating from either the planetary ionosphere or the solar wind (including solar wind $\mathrm{He}^{++}$) whereas Vasyliunas cycle return flow would include ions from internal sources (e.g., water group ions $\left(\mathrm{W}^{+}\right)$from Enceladus and $\mathrm{H}_{2}^{+}$from Titan [Thomsen et al., 2010]). However, heavy ions are preferentially lost down the magnetotail through Vasyliunastype tail reconnection (compared to lighter ions), which must also be included in this picture. Badman and Cowley [2007] also predicted that the flows would form layers a few Saturn radii $\left(R_{S}\right)$ wide in the vicinity of the post-dawn magnetopause, with the "Dungey layer" closer to the magnetopause and the "Vasyliunas layer" closer to the planet.

[7] Data taken by instruments mounted on the Cassini orbiter spacecraft have revealed evidence for reconnection in Saturn's magnetotail [Mitchell et al., 2005, 2009; Jackman et al., 2007, 2008; Hill et al., 2008], and it has been suggested that these events can be triggered by rapid compression of the system by the solar wind [Bunce et al., 2005]. Ion composition measurements made during some of the tail reconnection events reveal that the flows were rich in $\mathrm{W}^{+}$ ions, suggesting Vasyliunas-type tail reconnection [Hill et al., 2008]. 


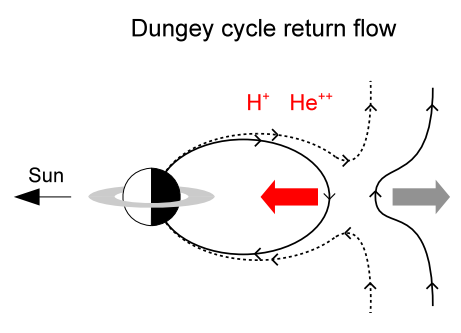

Vasyliunas cycle return flow

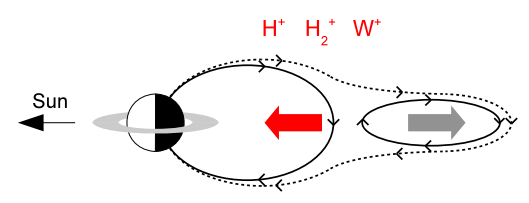

Figure 1. Schematics illustrating return flows resulting from Vasyliunas-type and Dungey-type reconnection in Saturn's magnetotail. Dashed and solid lines indicate magnetic field lines before and after reconnection, respectively. Return flows and their predicted ion compositions are shown as red block arrows and red text, respectively. The schematics are not to scale.

[8] In this paper we use ion data taken by the Cassini spacecraft in Saturn's post-dawn magnetosphere to identify an encounter with return flow from reconnection in the magnetotail. The plasma composition of the identified flow is consistent with Vasyliunas cycle return flow. The flow was supercorotating, which is highly atypical of Saturn's outer magnetosphere.

\section{Examining Plasma Flow in Saturn's Post-Dawn Magnetosphere}

[9] We surveyed data taken by the ion mass spectrometer (IMS) of the Cassini plasma spectrometer (CAPS) between July 2004 and March 2006, when the spacecraft explored Saturn's post-dawn magnetosphere at low-latitudes. Our region of interest was the outer magnetosphere, in the vicinity of the magnetopause, where the return flows are predicted to be [Badman and Cowley, 2007]. This region is typically between $\sim 20$ and $\sim 40 \mathrm{R}_{\mathrm{S}}$ from the planet.

[10] IMS measures ions with energy-per-charge between $1 \mathrm{~V}$ and $50 \mathrm{kV}$, with mass-per-charge $(\mathrm{m} / \mathrm{q})$ resolution. Since the spacecraft is three-axis stabilized the CAPS instrument is mounted on an actuator platform that rotates the instrument in order to improve the sensor fields-of-view (FOVs). IMS observes three main ion species in Saturn's magnetosphere: $\mathrm{H}^{+}(\mathrm{m} / \mathrm{q}=1), \mathrm{H}_{2}^{+}$or $\mathrm{He}^{++}(\mathrm{m} / \mathrm{q}=2)$, and $\mathrm{W}^{+}$(comprising $\mathrm{O}^{+}$, $\mathrm{OH}^{+}, \mathrm{H}_{2} \mathrm{O}^{+}$, and $\mathrm{H}_{3} \mathrm{O}^{+}$, with $\mathrm{m} / \mathrm{q}$ from 16 to 19). Accurate moments for each resolved species can only be derived from IMS data when the bulk flow is in the fan-like sensor FOV. Further details of the CAPS instrument and moment derivation have been described in detail by Young et al. [2004] and Thomsen et al. [2010], respectively.

[11] The radial distance of the spacecraft from Saturn does not give the position of the spacecraft in the outer magnetosphere because the position of Saturn's magnetopause is highly variable [e.g., Kanani et al., 2010], and L-shells at these distances are undefined. To isolate IMS data taken in the outer magnetosphere we considered 1.5-day intervals before (after) the first (last) magnetopause crossing of the outbound (inbound) pass of a spacecraft orbit. After Thomsen et al. [2010], we excluded moments where CAPS was not actuating, or the spacecraft was rolling, or the expected corotation direction was not in the IMS FOV, and we also excluded unreliable values as indicated by the moment calculation routine.

[12] This produced four orbit passes where reliable ion moments fall within the 1.5-day interval. In the mission nomenclature these are the inbound passes of the Revolution (Rev) A (October 2004), B (December 2004), 16 (October 2005), and 21 (February 2006) orbits. The spacecraft trajectory for each 1.5-day interval is shown in Figure 2. These were all low-latitude passes, within $5^{\circ}$ of the equatorial plane.

[13] Selected ion moments for each pass are shown in Figure 3. The $x$ axis common to all the panels is the time since the final magnetopause crossing of each pass, which is a proxy for the distance between the spacecraft and the magnetopause, and thus its position in the outer magnetosphere. Figure 3a shows $\mathrm{H}^{+}$number densities, Figure $3 \mathrm{~b}$ shows $\mathrm{H}^{+}$ temperatures, Figure $3 \mathrm{c}$ shows the azimuthal component of the $\mathrm{H}^{+}$flow velocity (the corotation component), and Figure $3 \mathrm{~d}$ shows the same flow component divided by the local speed of corotation with the planet. The similar data gap for each pass is primarily due to the flow not being in the IMS FOV due to the attitude of the spacecraft. The low azimuthal flow components close to the magnetopause may be due to boundary layer flows, which are not relevant to the present study.

[14] $\mathrm{H}^{+}$moments are shown in Figure 3 rather than one of the other two species because the $\mathrm{H}^{+}$number densities were consistently highest in the outer magnetosphere, and other

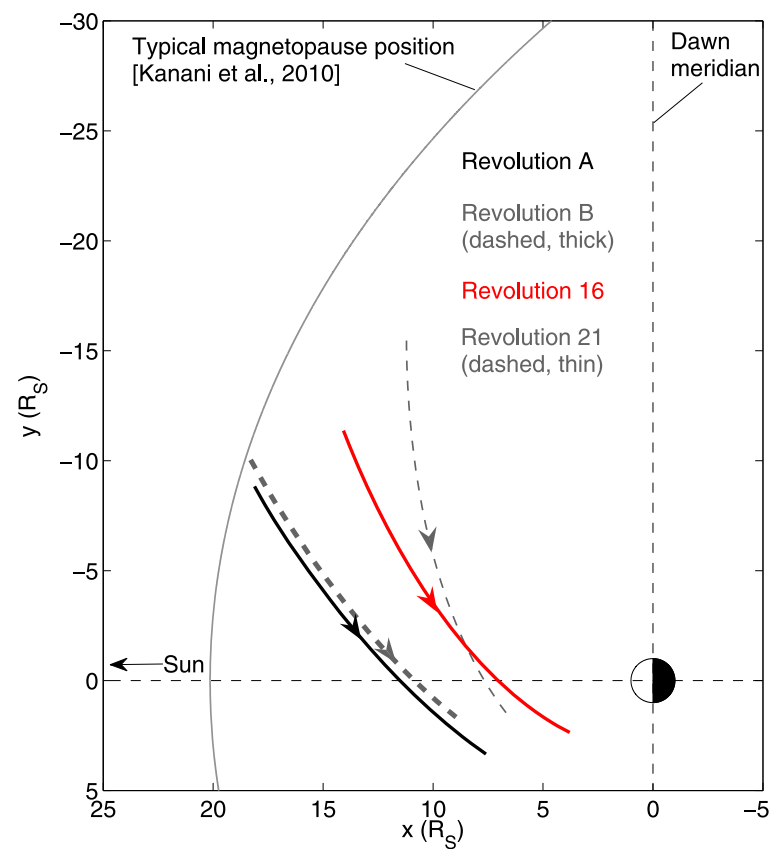

Figure 2. Trajectory of the Cassini spacecraft projected onto the equatorial plane following the final magnetopause crossing of the inbound passes of four orbits. 


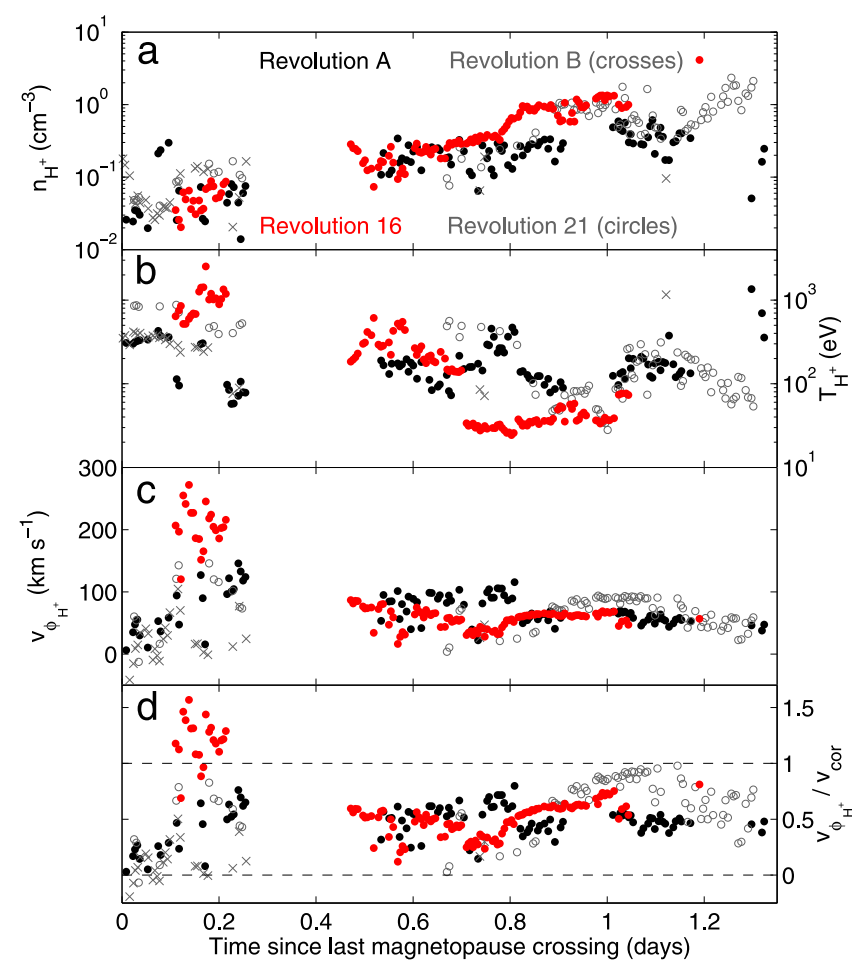

Figure 3. Ion moments derived from Cassini ion mass spectrometer data during the inbound passes of four orbits.

species were not resolved as frequently. In their survey of Cassini ion moments, Thomsen et al. [2010] showed that the velocity determinations for the light ion species are generally less reliable than for $\mathrm{W}^{+}$, which can affect the derived ion temperatures. This issue does not affect any of the moments presented in this paper. To demonstrate this, $\mathrm{W}^{+}$velocities are compared to $\mathrm{H}^{+}$velocities in the auxiliary material attached to this article. ${ }^{1}$

[15] Our understanding of return flow from the Dungey and Vasyliunas cycles suggests that the ion temperatures of both flows should be a few keV [Badman and Cowley, 2007]. The inbound pass of the Rev 16 orbit (shown in red) is a clear example of such heated flow in Saturn's outer magnetosphere (between $\sim 0.1$ and $\sim 0.2$ days), leading us to identify it as an example of return flow. This event also stands out as it had a flow speed that was $\sim 120 \%$ of corotation (supercorotation). This is highly atypical of Saturn's outer magnetosphere, where the flow is generally well below corotation [e.g., Thomsen et al., 2010]. In the next section we examine this event in more detail.

\section{Evidence for Return Flow on $\mathbf{1 0}$ October 2005}

[16] The return flow event on the Rev 16 inbound pass was observed by Cassini on 10 October 2005. Figure 4 shows data taken during an interval surrounding the event. Cassini was inbound, at a range of $\sim 17.5 \mathrm{R}_{\mathrm{S}}$, a dipole magnetic latitude of $\sim-0.3^{\circ}$, and $\sim 09: 40$ Saturn local time (SLT) (see Figure 2). The Saturn-centered spherical polar coordinate system used

\footnotetext{
${ }^{1}$ Auxiliary materials are available in the HTML. doi:10.1029/ 2010GL046149.
}

in Figure 4 is Kronocentric R/Theta/Phi (KRTP) coordinates, where the $r$ axis points away from Saturn, the $\theta$ axis points southward, and the $\varphi$ axis points in the corotation direction.

[17] Figure 4a shows magnetic field data taken by the fluxgate magnetometer of the Cassini magnetic field investigation [Dougherty et al., 2004]. Figures $4 \mathrm{~b}$ and $4 \mathrm{c}$ show the number densities and temperatures of all the ion species (resolved by IMS) and of the electrons (resolved by the CAPS electron spectrometer [Young et al., 2004; Lewis et al., 2008]), respectively. Figure 4d shows the components of the $\mathrm{H}^{+}$bulk flow vectors, with the local corotation speed shown as a dotted black line). The dark-gray-shaded intervals indicate when Cassini was outside the magnetosphere, and the light-gray-shaded interval indicates the return flow event. We refer the reader to the auxiliary material attached to this article for a discussion of why IMS moments could only be derived for part of this interval, and to see the spectra that are the basis of the presented ion and electron moments.

[18] Throughout the subject interval the magnetic field remained predominantly southward. During the return flow event all three of the main ion species were resolved by IMS. $\mathrm{W}^{+}$ions were present, and the ratio of the number density of the ion species with $\mathrm{m} / \mathrm{q}=2$ to the number density of $\mathrm{H}^{+}$was $\sim 0.5$ throughout the event. Since this ratio is typically 0.05 in the solar wind (where the $\mathrm{m} / \mathrm{q}=2$ species is $\mathrm{He}^{++}$), this higher ratio leads us to identify the $\mathrm{m} / \mathrm{q}=2$ species as $\mathrm{H}_{2}^{+}$ [Thomsen et al., 2010]. The temperatures of all the species were of the order of $1 \mathrm{keV}$, with heavier species being hotter, and all ion species were hotter than the electrons. We note that the return flow event can be separated into two sub-intervals on the basis of the $\mathrm{W}^{+}$number density, which was significantly lower towards the end of the event. The ion moments after 21:00 reveal a subcorotating plasma environment that was far colder than that observed earlier in the day.

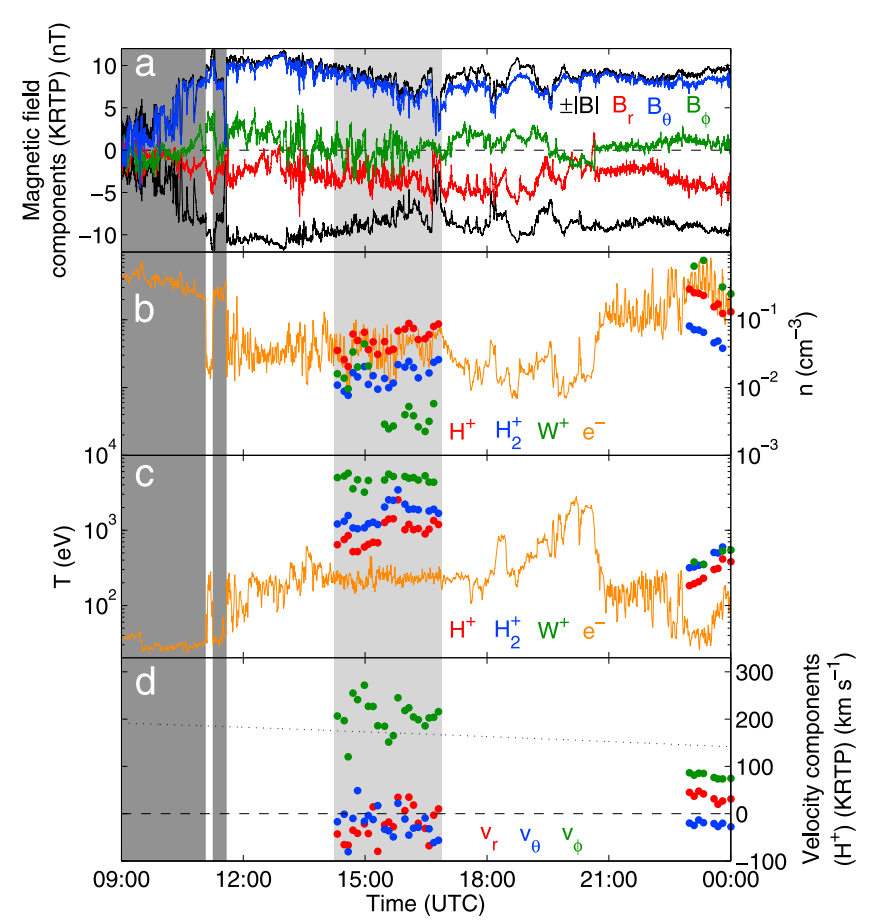

Figure 4. Data taken by Cassini on 10 October 2005 . 
[19] The ion composition measured during this event suggests that it resulted from magnetotail reconnection involving closed field lines, consistent with the Vasyliunas cycle (see Figure 1). The ion temperatures and densities, are in agreement with the predictions of Badman and Cowley [2007] for return flow. Assuming that the magnetosphere was not expanding or contracting when the spacecraft traversed the return flow region, we estimate the spatial scale of the region as $\sim 1 R_{S}$. Although the scale of the return flow region remains unclear, we note that this lower limit is of the same order as that predicted by Badman and Cowley [2007].

\section{Discussion}

[20] We have presented evidence for supercorotating return flow from reconnection in Saturn's magnetotail, based on ion data taken by the Cassini spacecraft in the post-dawn magnetosphere. This return flow was identified on the basis of elevated ion temperatures in the outer magnetosphere, and the plasma composition of the flow suggests that it resulted from reconnection of closed field lines in the magnetotail, consistent with the Vasyliunas cycle.

[21] The spacecraft only encountered clear return flow on one of the four passes we were able to examine. Ion temperatures measured during the other passes in a similar region were not of order $1 \mathrm{keV}$ (indicative of return flow [Badman and Cowley, 2007]), although they approached such values during Rev 21 (see Figure 3), implying there may also have been return flow on this pass. No passes were associated with a solar wind-like composition consistent with Dungey cycle return flow (see Figure 1). Due to the limited coverage of Cassini ion moments with appropriate viewing we cannot draw firm conclusions regarding either the presence of return flows on the other passes, or the existence of Dungey cycle return flows. Return flow like that discussed in Section 3 may be subject to strong temporal variations.

[22] It is difficult to address whether the clear example of return flow was mass-depleted due to the lack of information about the plasma density throughout the system, and how it changed with time. Comparing ion densities measured during the return flow (see Figure 4) with typical densities at this radial distance in Saturn's magnetosphere [Thomsen et al., 2010] suggests that the densities of the light-ion species were comparable to or less than typical values, and the $\mathrm{W}^{+}$ densities were a factor of $\sim 3$ lower than the average. However, the average values are based on radial distance from the planet, which is not an accurate measure of the spacecraft position in the outer magnetosphere, as discussed in Section 2. The comparison with the densities measured in a similar region during the other three passes is also unreliable, due to the level of plasma variability in Saturn's outer magnetosphere [e.g., Thomsen et al., 2010]. It is unclear whether the return flow was mass depleted.

[23] The presence of a clear return flow on only one of the four passes and the supercorotation of the observed flow may be due to solar wind compression of the magnetosphere. A proxy for magnetospheric size is the magnetopause standoff distance, which can be calculated for the final magnetopause crossing of each pass using Kanani et al.'s [2010] model of Saturn's magnetopause. The standoff distance for Rev 16 was $16.9 \mathrm{R}_{\mathrm{S}}$, whereas for Rev A, B, and 21 it was 20.2, 20.7, and $16.2 \mathrm{R}_{\mathrm{S}}$ respectively. Although these values are only 'snapshots' of the size of the system they suggest that the return flow was observed under more compressed conditions, as was the potential return flow on Rev 21.

[24] Compressed conditions are expected to promote the operation of both the Dungey and Vasyliunas cycles, by enhancing dayside reconnection [e.g., Badman and Cowley, 2007] and promoting tail reconnection [e.g., Bunce et al., 2005]. Compression of the outer magnetosphere is also expected to increase corotational flow speeds by conservation of angular momentum as flux tubes are pushed closer to the planet [Sittler et al., 2006]. Solar wind compression could explain why clear return flow was not observed on all the passes, and why the flow during the example was supercorotating. Supercorotating flow in this region of Saturn's magnetosphere was predicted by Talboys et al. [2009a] as the cause of leading high-latitude magnetic field signatures [Talboys et al., 2009a, 2009b]. Such leading fields border the main auroral current system, and do not appear to be a permanent magnetic feature. As in previous studies [e.g., Galopeau and Lecacheux, 2000], we suggest that plasma flow in Saturn's outer magnetosphere strongly influences planetary auroral emissions.

[25] Acknowledgments. AM acknowledges useful discussions with A. P. Walsh, C. F. Forsyth, and N. Achilleos. We acknowledge the support of the CAPS and MAG data processing/distribution staff, and L. K. Gilbert and G. R. Lewis for Cassini ELS data processing. This work was supported by UK STFC through rolling grants to MSSL/UCL and Imperial College London, and an STFC Postdoctoral Fellowship awarded to CSA. Work at Los Alamos was conducted under the auspices of the U.S. Department of Energy, with support from NASA's Cassini program. DTY acknowledges support of JPL contract 1356497 with SwRI.

\section{References}

Badman, S. V., and S. W. H. Cowley (2007), Significance of Dungeycycle flows in Jupiter's and Saturn's magnetospheres, and their identification on closed equatorial field lines, Ann. Geophys., 25, 941-951, doi:10.5194/angeo-25-941-2007.

Bunce, E. J., S. W. H. Cowley, D. M. Wright, A. J. Coates, M. K. Dougherty, N. Krupp, W. S. Kurth, and A. M. Rymer (2005), In situ observations of a solar wind compression-induced hot plasma injection in Saturn's tail, Geophys. Res. Lett., 32, L20S04, doi:10.1029/ 2005 GL022888.

Dougherty, M. K., et al. (2004), The Cassini magnetic field investigation, Space Sci. Rev., 114(1-4), 331-383, doi:10.1007/s11214-004-1432-2.

Dungey, J. W. (1961), Interplanetary magnetic field and the auroral zones, Phys. Rev. Lett., 6, 47-48, doi:10.1103/PhysRevLett.6.47.

Galopeau, P. H. M., and A. Lecacheux (2000), Variations of Saturn's radio rotation period measured at kilometer wavelengths, J. Geophys. Res., 105, 13,089-13,101, doi:10.1029/1999JA005089.

Hill, T. W., et al. (2008), Plasmoids in Saturn's magnetotail, J. Geophys. Res., 113, A01214, doi:10.1029/2007JA012626.

Jackman, C. M., C. T. Russell, D. J. Southwood, C. S. Arridge, N. Achilleos, and M. K. Dougherty (2007), Strong rapid dipolarizations in Saturn's magnetotail: In situ evidence of reconnection, Geophys. Res. Lett., 34, L11203, doi:10.1029/2007GL029764.

Jackman, C. M., et al. (2008), A multi-instrument view of tail reconnection at Saturn, J. Geophys. Res., 113, A11213, doi:10.1029/2008JA013592.

Kanani, S. J., et al. (2010), A new form of Saturn's magnetopause using a dynamic pressure balance model, based on in situ, multi-instrument Cassini measurements, J. Geophys. Res., 115, A06207, doi:10.1029/ 2009JA014262.

Lewis, G. R., et al. (2008), Derivation of density and temperature from the Cassini-Huygens CAPS electron spectrometer, Planet. Space Sci., 56(7), 901-912, doi:10.1016/j.pss.2007.12.017.

Mitchell, D. G., et al. (2005), Energetic ion acceleration in Saturn's magnetotail: Substorms at Saturn?, Geophys. Res. Lett., 32, L20S01, doi:10.1029/2005GL022647.

Mitchell, D. G., et al. (2009), Recurrent energization of plasma in the midnight-to-dawn quadrant of Saturn's magnetosphere, and its relationship to auroral UV and radio emissions, Planet. Space Sci., 57, 17321742, doi:10.1016/j.pss.2009.04.002. 
Sittler, E. C., Jr., M. F. Blanc, and J. D. Richardson (2006), Proposed model for Saturn's auroral response to the solar wind: Centrifugal instability model, J. Geophys. Res., 111, A06208, doi:10.1029/ 2005JA011191.

Talboys, D. L., C. S. Arridge, E. J. Bunce, A. J. Coates, S. W. H. Cowley, and M. K. Dougherty (2009a), Characterization of auroral current systems in Saturn's magnetosphere: High-latitude Cassini observations, J. Geophys. Res., 114, A06220, doi:10.1029/2008JA013846.

Talboys, D. L., C. S. Arridge, E. J. Bunce, A. J. Coates, S. W. H. Cowley, M. K. Dougherty, and K. K. Khurana (2009b), Signatures of fieldaligned currents in Saturn's nightside magnetosphere, Geophys. Res. Lett., 36, L19107, doi:10.1029/2009GL039867.

Thomsen, M. F., D. B. Reisenfeld, D. M. Delapp, R. L. Tokar, D. T. Young, F. J. Crary, E. C. Sittler, M. A. McGraw, and J. D. Williams (2010), Survey of ion plasma parameters in Saturn's magnetosphere, J. Geophys. Res., 115, A10220, doi:10.1029/2010JA015267.

Vasyliunas, V. M. (1983), Plasma distribution and flow, in Physics of the Jovian Magnetosphere, edited by A. J. Dessler, chap. 11, pp. 395-453, Cambridge Univ. Press, New York.
Young, D. T., et al. (2004), Cassini plasma spectrometer investigation, Space Sci. Rev., 114(1-4), 1-112, doi:10.1007/s11214-004-1406-4.

C. S. Arridge, A. J. Coates, and A. Masters, Mullard Space Science Laboratory, Department of Space and Climate Physics, University College London, Holmbury St. Mary, Dorking RH5 6NT, UK. (am2@ mssl.ucl.ac.uk)

S. V. Badman, JAXA Institute of Space and Aeronautical Science, 3-1-1 Yoshinodai, Chuo-ku, Sagamihara, Kanagawa 252-5210, Japan.

M. K. Dougherty, Space and Atmospheric Physics Group, Blackett Laboratory, Imperial College London, Prince Consort Road, London SW7 2AZ, UK

M. F. Thomsen, Space Science and Applications, Los Alamos National Laboratory, MS D466, Los Alamos, NM 87545, USA.

D. T. Young, Space Science and Engineering Division, Southwest Research Institute, 6220 Culebra Rd., PO Drawer 28510, San Antonio, TX 78228, USA. 